\title{
Effect of sleeping alone on sleep quality in female bed partners of snorers
}

\author{
M. Blumen*,\#, , M.A. Quera Salva*,\#, M-P. d'Ortho ${ }^{+}$, K. Leroux ${ }^{\S}$, P. Audibert ${ }^{\S}$, \\ C. Fermanian*, ${ }^{*}$, F. Chabolle ${ }^{f}$ and F. Lofaso*,\#
}

ABSTRACT: The aim of the present study was to objectively measure the effect of sleeping alone for one night on sleep quality in female bed partners of male snorers.

Females complaining of poor sleep due to snoring by their bed partner and having no known hearing loss or snoring were included in a prospective multicentre cross-sectional study. 23 females underwent one polysomnography recording while sleeping with their bed partner and another while sleeping alone. Their sleep parameters were compared between the two nights.

We excluded seven couples because the female partner snored for $>10 \%$ of the sleep time $(n=6)$ or had obstructive sleep apnoea syndrome $(n=1)$. In the remaining 16 females, sleep time, sleep efficiency, arousal index and percentages of deep sleep (stages 3-4) and rapid eye movement (REM) sleep were not significantly different between the two nights. Percentages of light sleep (non-REM stage 2) and awakening index were lower when sleeping alone $(p=0.023$ and $p=0.046$, respectively).

Sleep quality was decreased and sleep fragmentation increased in females sleeping with male snorers. Some females had unrecognised snoring. However, our data do not suggest that objective sleep quality improves substantially in the female nonsnoring partner when she sleeps alone for one night.

\section{KEYWORDS: Bed partner, sleep apnoea syndrome, snoring}

$(8)$ leeping with a snoring partner has been reported to increase daytime feelings of stress, depression [1] and fatigue [2], although there were no consistent changes in quality of life evaluated through questionnaires $[3,4]$ or in daytime sleepiness [4, 5]. Poor sleep quality has been reported by more than twothirds of bed partners of snorers [3]. These subjective complaints were related not only to snoring, but also to apnoeas and restlessness [3].

Nonsnoring bed partners may try various methods to improve their sleep quality, such as using ear plugs or sleeping pills, or both [6]. Treatment of the snorer may bring relief to the partner. A few studies evaluated the sleep quality of those with bed partners whose sleep apnoea syndrome (SAS) was treated with continuous positive airway pressure (CPAP) $[1,3,4,7,8]$. Improvements were noted in the nonsnoring partners' daytime sleepiness scores (which were within the normal range at baseline) and in anxiety scores, but not in depression scores, after 6-8 weeks of CPAP [1]. In one of the few studies supplying objective data, CPAP during the second half of the study night was associated with a decreased arousal index and increased sleep efficiency in the partners [8]. In a cross-over study of CPAP versus placebo for 4 weeks each, CPAP was associated with better subjective sleep quality in the partner, but no improvements were noted in quality of life or objective sleep parameters (sleep efficiency, arousal index, sleep latency, time in slow wave sleep (SWS) and time in rapid eye movement (REM) sleep) [3].

Some couples decide to sleep in separate rooms in order to improve their sleep quality. Among patients referred for snoring or suspected obstructive SAS and scheduled for surgical treatment of nasal obstruction, $40 \%$ had partners who slept in a separate room at least once a week [6], and in a population of SAS patients referred for initiation of CPAP therapy, $18 \%$ had partners who did not share their bed on a regular basis; however, these partners had worse anxiety and depression scores at baseline than the bedsharing partners [1]. In addition, in a recent study in a nonclinical population, having a bed partner with sleep-disordered breathing was not associated with poorer sleep quality than having a bed partner without sleep-disordered breathing [9]. Finally, there is no objective evidence that sleeping alone results in better sleep among

\section{AFFILIATIONS}

*Physiology and Functional Testing, AP-HP, Raymond Poincaré Teaching Hospital, Université Versailles-St Quentin en Yvelines, Garches, "Technological Innovations Centre, Garches,

'Centre Médical Veille-Sommeil, Paris,

+Physiology and Functional Testing, AP-HP, Bichat-Claude Bernard Teaching Hospital, Paris,

${ }^{\S}$ Association d'Entraide des Polios et Handicapés (ADEP Assistance),

Puteaux, and

${ }^{f}$ Foch Teaching Hospital, Université Versailles-St Quentin en Yvelines,

Suresnes, France.

CORRESPONDENCE

M. Blumen

Sleep unit

Raymond Poincaré Teaching Hospital 104 Boulevard Raymond Poincaré 92380 Garches

France

E-mail: mbjblu@club-internet.fr

Received:

Jan 232009

Accepted after revision:

May 312009

First published online:

July 022009 
individuals consulting with the bed-partner for his/her snoring and reporting that this snoring is bothersome.

The goal of this study was to obtain objective sleep parameters in female bed partners of male snorers during two nights at home in the usual bedroom, one with and one without the bed partner.

\section{MATERIALS AND METHODS}

We performed a prospective multicentre study. The appropriate ethics committee approved the study, and all participants gave their written informed consent prior to study inclusion.

\section{Study participants}

Participants were recruited prospectively at sleep units in three hospitals and one private centre in and around Paris, France from April 2003 to August 2007. All participants were females with a male bed partner whose snoring they described as bothersome. Participants underwent a preliminary ambulatory sleep study to evaluate chronic snoring, particularly regarding the possible presence of SAS, defined as an apnoea/hypopnoea index (AHI) $>10 \mathrm{~h}^{-1}$. The partners, one male and one female, had been in a relationship for $\geqslant 1 \mathrm{yr}$ long that was stable and described by both partners as good. The female partners complained of poor sleep quality due to their bed partner's chronic snoring and were willing to undergo two sleep studies. A room available at home where the snorer could sleep separately from his partner was required, along with an absence of any plan to relocate to another geographic area within the next 6 months. We did not include couples in which the snorer was a professional driver or had excessive daytime sleepiness (Epworth score >10), known cardiovascular disease or chronic alcoholism. These exclusion criteria were chosen for ethical and legal reasons: our study might have delayed treatment, thereby increasing the risk of motor vehicle accidents or cardiovascular disease in the individuals with snoring or SAS. Also ineligible were females who were known to snore and who complained of poor sleep quality due to their own sleep disorders (e.g. insomnia or restless leg syndrome), used sleeping pills or had marked hearing loss. Finally, we did not include couples who did not usually share the same bed.

\section{Study measurements}

No treatment for snoring or sleep apnoea was initiated before study completion. Snoring volume was evaluated subjectively by the female partner using a visual analog scale from 0 (no snoring) to 10 (snoring heard from another room) before the first sleep study. The female partner underwent two overnight sleep studies, at home, within 1 week of each other. The couple was asked to keep regular bedtimes for at least a week before the study nights. The female partner was recorded while sleeping in the bedroom with her male partner during one night and while sleeping alone in the same bedroom during the other night. Ventilation was recorded in the male partner during the shared-bed night and in the female partner during the night spent alone. For each sleep study, a technician went to the couple's home to place the electrodes several hours before the couple's usual bedtime, thus allowing habituation of the participants to the recording equipment. The recording equipment was removed by the study participants themselves the next morning. Polysomnography recordings were obtained using a CID 102/108 device (Cidelec, Sainte Gemmes sur Loire, France) and included two electroencephalogram leads (C3-A2, C4-A1), two electro-oculogram leads, and one chin electromyogram lead. The ventilation study consisted of recording snoring (via a microphone placed on the neck just over the trachea), nasal flow (through a nasal cannula), thoracoabdominal movements (through strain gauge belts), oximetry and heart rate.

The ventilatory and sleep traces were evaluated separately by observers who were blinded to the sleep condition (alone or with the partner). Apnoea was defined as a nasal flow decrease of $\geqslant 90 \%$ and hypopnoea as a nasal flow decrease $>50 \%$ or in the $30 \%-50 \%$ range and accompanied with a $\geqslant 3 \%$ fall in oxygen saturation. Sleep stages were scored manually according to ReCHTSCHAFFEN and KALES [10] on 30-s epochs. Arousals were defined following American Sleep Disorders Academy criteria [11]. The following ventilatory parameters were determined: AHI (number of apnoeas and hypopnoeas per hour of sleep), presence or absence of snoring, snoring time, snoring index (number of snores per hour) and mean snoring volume. Snoring was defined by the manufacturer as a sound with a frequency within the $20-200 \mathrm{~Hz}$ range and a volume in the microphone chamber $>76 \mathrm{~dB}$. We arbitrarily defined snoring as snoring sounds for $>10 \%$ of the total sleep time. Sleep quality was assessed based on the following parameters: total sleep time; sleep efficiency; wake time after sleep onset (WASO); arousal index (number of arousals per hour of total sleep time); number of awakenings per hour of sleep (or awakening index); and time and percentage of total sleep time spent in light sleep (stages 1 and 2), SWS (stages 3 and 4), and REM sleep.

\section{Statistics}

The order of the two nights (with then without the partner or without then with the partner) in each female was randomly assigned using a permuted block design [12].

Mean \pm SD values were computed to describe the study population. Wilcoxon's nonparametric tests and paired t-tests were used to compare sleep quality parameters in the female partner between the night spent alone and the night with the male partner. We used the nonparametric Mann-Whitney and t-test test to compare sleep-quality parameters between female partners of males with and without SAS [13]. Univariate repeated-measures ANOVA was used to evaluate interactions between the sleeping condition (with the partner or alone) and the order of the two nights [14]. A p-value $<0.05$ was considered significant.

\section{RESULTS}

From April 2003 to August 2007, 30 couples who met the inclusion criteria were identified. Seven couples were not included, either because of busy work schedules $(n=3)$ or because the snorer had severe SAS requiring prompt treatment initiation $(n=4)$. Of the 23 remaining couples, 16 were married. All female partners of the snorers complained of sleep disturbance due to their partner's snoring, but no evidence of severe tension was noted or reported by the couples. Neither the snorers nor their female partners reported highly variable sleeping durations or bedtimes; in particular, none worked on night shifts. Of the 23 couples, seven $(30.4 \%)$ were excluded 


\begin{tabular}{lcc}
\hline TABLE 1 & $\begin{array}{l}\text { Clinical features of the } 16 \text { couples included in the } \\
\text { study }\end{array}$ \\
& Male snorer & Female nonsnorer \\
\hline Subjects $\mathbf{n}$ & 16 & 16 \\
Age yrs & $45.3 \pm 10.6$ & $44.6 \pm 11.8$ \\
BMI $\mathbf{~ k g} \cdot \mathbf{m}^{-2}$ & $26.5 \pm 3.9$ & $21.7 \pm 3.5$ \\
Epworth score & & $6.5 \pm 4.4$ \\
AHI events $\mathbf{h}^{-1}$ & & $2.6 \pm 3.6$ \\
In 16 females & & \\
In 7 males without SAS & $2.3 \pm 2.7$ & \\
In 9 males with SAS & $34.9 \pm 20.3$ & \\
\hline
\end{tabular}

Data are expressed as mean $\pm \mathrm{SD}$, unless otherwise stated. BMI: body mass index; AHI: apnoea/hypopnoea index; SAS: sleep apnoea syndrome.

because the recordings showed snoring in the female partner for $>10 \%$ of the total sleep time; of these seven females, one had SAS. In these seven excluded couples, five male partners snored for $<10 \%$ of the total sleep time and three female partners had longer snoring periods than their male partners.

The main features in the 16 included couples are listed in table 1. Among the males, nine had SAS. Snoring parameters are described in table 2.

Table 3 compares the sleep parameters in the females during the nights with and without the male partners. Two parameters were significantly better when the female partners slept alone, namely, the time spent in non-REM stage 2 sleep and the awakening index. Total sleep time, sleep efficiency, sleep latencies, WASO and the arousal index were not significantly different between the two nights. During the night with the male partner, awakenings and arousals were unrelated to snoring initiation or exacerbation. Table 4 , which compares the sleep parameters in the females between the two orders of recording nights, shows that the order effect was not significant for any of the parameters.

The mean time interval between the two nights was 6.2 days in the "alone then with partner" group and 7.0 days in the "with partner then alone" group (Wilcoxon's two-sample test: $\mathrm{p}=0.91$; t-test: $\mathrm{p}=0.72$; nonsignificant). The mean \pm SD interval overall was $6.75 \pm 3.97$ days. The interaction between sleeping condition and order was not significant $(p \geqslant 0.30$ for all parameters).

No significant differences in sleep parameters were found between female partners of males with and without SAS.

\section{DISCUSSION}

We found no clear evidence that sleeping alone for one night substantially improved sleep quality. Only the time spent in light sleep (non-REM stage 2) and the awakening index improved significantly when the female partners slept alone; however, the differences were small and probably of limited clinical relevance. SWS was decreased under both conditions [15], whereas no increase in sleep fragmentation or decrease in REM sleep was observed [15]. The absence of increased sleep fragmentation and the fact that awakenings were unrelated to

\section{TABLE 2 Snoring parameters in the 16 males}

\begin{tabular}{lc} 
Parameters & \\
\hline & \\
VAS snoring score & $7.5 \pm 2.7$ \\
Mean snoring volume dB & $88.6 \pm 4.2$ \\
Total snores $\mathbf{n}$ & $1172 \pm 1109$ \\
Snoring episodes $\mathbf{n}$ & $72.5 \pm 76.0$ \\
Snoring index & $168 \pm 165$ \\
Snoring duration min & $89.8 \pm 83.7$ \\
Per cent of sleep time spent snoring & $21.1 \pm 20.8$
\end{tabular}

Data are expressed as mean \pm SD. VAS: 10-point visual analog scale evaluated by the female bed partner.

snoring initiation or exacerbation suggest that the snoring volume was not sufficient to cause awakening. Furthermore, habituation to noise may have occurred [16]. Sleeping alone for one night may not be sufficient to allow recovery of SWS in individuals chronically exposed to snoring at night. A longer period of sleeping in quiet surroundings may be needed to restore the normal sleep structure. Conversely, a rebound effect might occur if the snoring was the main cause of SWS deficiency.

Our study has several limitations. First, our sample was small (16 couples), and the standard deviations were large for some of the study variables. A larger sample might have led to the identification of additional significant differences. Nevertheless, our results indicate that sleeping without the snoring bed partner for one night did not normalise the sleep of the nonsnoring bed partner. A larger sample might enable the detection of small differences, but these might well be of no clinical significance.

Secondly, we did not habituate our subjects to the equipment during an entire night and we did not evaluate night-to-night variability. In addition, the female partners wore thoracic and abdominal belts, a nasal cannula and a microphone when sleeping alone but not when sleeping with the male partners. Sleep disturbances induced by these items of equipment may have masked improvements in sleep quality induced by sleeping alone. However, when the two partners slept together, they were connected to the same recording box and had multiple wires in the bed. We believe that the resulting discomfort was probably similar to that experienced by the female partner when she slept alone wearing a nasal cannula and thoraco-abdominal belts. Moreover, it has been shown that recordings performed at home are not associated with a firstnight effect or night-to-night variability [17].

Thirdly, sleeping alone for one night may not be sufficient to allow recovery of sleep quality in individuals who are chronically exposed to noise at night. In the female partners, some of the sleep parameters, such as the arousal index and deep sleep duration, were abnormal [18]. Conceivably, chronic exposure to noise at night may lead to alterations in sleep structure that cannot be eliminated by a single quiet night. Data on the effect of CPAP in the nonsnoring bed partner suggest a larger effect after $\geqslant 6$ weeks [1] than after $<1$ month [3]. 
TABLE 3 Comparison of sleep parameters in the female partners during the nights with and without the male partners

\begin{tabular}{|c|c|c|c|c|}
\hline & Night alone & $\begin{array}{l}\text { Night with } \\
\text { partner }\end{array}$ & $\begin{array}{c}\text { Nonparametric } \\
\text { p-value }\end{array}$ & $\begin{array}{c}\text { Parametric } \\
\text { p-value }\end{array}$ \\
\hline Sleep efficiency \% & $87.6 \pm 9.9$ & $83.4 \pm 10.2$ & 0.224 & 0.232 \\
\hline Stage 3 latency $\mathrm{min}$ & $22.1 \pm 30.9$ & $27.8 \pm 19.9$ & 0.245 & 0.524 \\
\hline REM sleep latency min & $84.0 \pm 31.9$ & $91.4 \pm 48.7$ & 0.717 & 0.622 \\
\hline Non-REM stage $3-4 \%$ & $16.8 \pm 6.5$ & $14.9 \pm 4.8$ & 0.147 & 0.195 \\
\hline Non-REM stage $3-4$ duration min & $59.1 \pm 24.7$ & $50.5 \pm 20.4$ & 0.217 & 0.235 \\
\hline REM sleep \% & $24.8 \pm 8.7$ & $21.4 \pm 7.8$ & 0.196 & 0.179 \\
\hline WASO min & $49.4 \pm 44.0$ & $64.3 \pm 44.3$ & 0.301 & 0.282 \\
\hline Awakening index & $4.0 \pm 1.7$ & $4.8 \pm 1.9$ & 0.046 & 0.086 \\
\hline Arousal index & $11.4 \pm 4.2$ & $11.8 \pm 5.1$ & 0.214 & 0.763 \\
\hline
\end{tabular}

Data are expressed as mean $\pm S D$, unless otherwise stated. REM: rapid eye movement; WASO: wake time after sleep onset.

A longer period of sleeping in quiet surroundings may be needed to restore normal sleep structure. Sleep disturbances due to chronic noise from night traffic improve over time but do not resolve completely [16]. Finally, sleeping in separate rooms may induce adverse psychological effects. In one study, the participants who slept apart had worse quality of life scores and higher anxiety and depression scores [1]. Nevertheless, in accordance with the small beneficial effects of sleeping alone in our study, a recent study in a nonclinical population failed to demonstrate that having a partner with sleep-disordered breathing was associated with worse sleep quality [9].
An unexpected finding from our study was that seven (30.4\%) of the female partners snored. Furthermore, their snoring occupied more of the total sleep time than did the snoring of their partners. In none of the couples was either partner aware that the female snored. Thus, the sleep disturbances reported by some female partners of male snorers may be due to their own sleep disturbances.

In conclusion, we found little objective evidence that sleeping apart for one night significantly improved objective sleep quality in the female partners of male snorers. Sleeping apart

TABLE 4 Comparison of sleep parameters between the two groups of female partners defined by the order of nights with and without the male partners

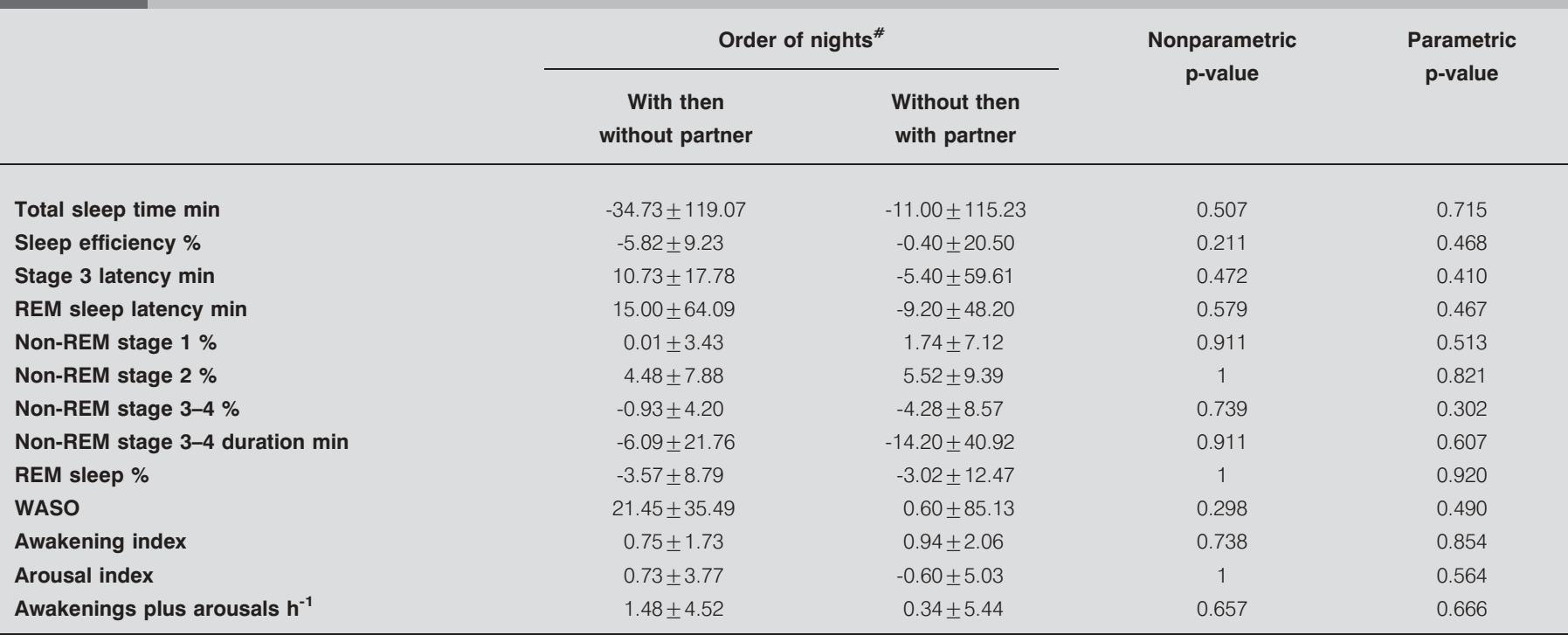

Data are expressed as mean $\pm \mathrm{SD}$, unless otherwise stated. REM: rapid eye movement; WASO: wake time after sleep onset. ${ }^{\#}$ : differences in sleep parameters, with partner minus without. 
may not be the best solution when a female is bothered by her bed partner's snoring. Neither does ignoring the problem seem appropriate, particularly when sleep apnoeas occur. Data in the literature suggest that treating the snoring/sleep apnoea may be the best approach. Our study indicates that sleep specialists must take care to ensure they treat the right person.

\section{SUPPORT STATEMENT}

Financial support was received from ADEP Assistance, Puteaux, France for renting the recording equipment.

\section{STATEMENT OF INTEREST}

None declared.

\section{REFERENCES}

1 Doherty LS, Kiely JL, Lawless G, et al. Impact of nasal continuous positive airway pressure therapy on the quality of life of bed partners of patients with obstructive sleep apnea syndrome. Chest 2003; 124: 2209-2214.

2 Ulfberg J, Carter N, Edling C. Sleep-disordered breathing and occupational accidents. Scand J Work Environ Health 2000; 26: 237-242.

3 McArdle N, Kingshott R, Engleman HM, et al. Partners of patients with sleep apnoea/hypopnoea syndrome: effect of CPAP treatment on sleep quality and quality of life. Thorax 2001; 56: 513-518.

4 Parish JM, Lyng PJ. Quality of life in bed partners of patients with obstructive sleep apnea or hypopnea after treatment with continuous positive airway pressure. Chest 2003; 124: 942-947.

5 Breugelmans JG, Ford DE, Smith PL, et al. Differences in patient and bed partner-assessed quality of life in sleep-disordered breathing. Am J Respir Crit Care Med 2004; 170: 547-552.

6 Virkkula P, Bachour A, Hytonen M, et al. Patient- and bed partnerreported symptoms, smoking, and nasal resistance in sleepdisordered breathing. Chest 2005; 128: 2176-2182.
7 Kiely JL, McNicholas WT. Bed partners' assessment of nasal continuous positive airway pressure therapy in obstructive sleep apnea. Chest 1997; 111: 1261-1265.

8 Beninati W, Harris CD, Herold DL, et al. The effect of snoring and obstructive sleep apnea on the sleep quality of bed partners. Mayo Clin Proc 1999; 74: 955-958.

9 Sharief I, Silva GE, Goodwin JL, et al. Effect of sleep disordered breathing on the sleep of bed partners in the Sleep Heart Health Study. Sleep 2008; 31: 1449-1456.

10 Rechtschaffen A, Kales A. A Manual of Standardized Terminology, Techniques, and Scoring Systems for Sleep Stages of Human Subjects. Washington, US Government Printing Office, 1968.

11 American Academy of Sleep Medicine. The AASM Manual for the Scoring of Sleep and Associated Events. Rules, Terminology and Technical Specifications. Westchester, American Academy of Sleep Medicine, 2007; p. 37.

12 European Agency for the Evaluation of Medical Products. Note for Guidance on Statistical Principles for Clinical Trials. ICH 9. London, European Agency for the Evaluation of Medical Products, 1998.

13 Conover WJ. Practical Nonparametric Statistics. 2nd Edn. New York, Wiley, 1980.

14 Winer B. Statistical Principles in Experimental Design. 2nd Edn. New York, McGraw-Hill, 1971.

15 Walsleben JA, Kapur VK, Newman AB, et al. Sleep and reported daytime sleepiness in normal subjects: the Sleep Heart Health Study. Sleep 2004; 27: 293-298.

16 Ouis D. Exposure to nocturnal road traffic noise: sleep disturbance and its after effects. Noise Health 1999; 1: 11-36.

17 Sharpley AL, Solomon RA, Cowen PJ. Sleep stability with home sleep recording and automatic sleep stage analysis. Sleep 1990; 13: 538-540.

18 Gosselin N, Michaud M, Carrier J, et al. Age difference in heart rate changes associated with micro-arousals in humans. Clin Neurophysiol 2002; 113: 1517-1521. 